\title{
Tumor de células de la granulosa juvenil de ovario. Presentación inusual. Reporte de un caso
}

\author{
Drs. Ernesto Lara, ${ }^{1}$ Franco Calderaro Di Ruggiero, ${ }^{2}$ Carmen Silva, ${ }^{3}$ Johatson Freytez. ${ }^{4}$
}

\begin{abstract}
RESUMEN
Los tumores de células de la granulosa representan el $2 \%$ de los tumores de ovario. Son clasificados en tipo adulto y juvenil. El juvenil está asociado a pseudopubertad precoz, menstruación irregular o síntomas inespecíficos como dolor abdominal y masa palpable. Más del $95 \%$ están confinados al ovario. Se describe el caso de una paciente de 31 años de edad, quien consultó por aumento de volumen abdominal y pérdida de peso de 6 meses de evolución. Se diagnosticó tumor de ovario derecho. Se practicó exéresis del tumor por laparotomía ginecológica con biopsia por corte congelado positiva y cirugía estadificadora. Anatomía patológica reportó tumor del estroma y cordones sexuales sugestivo de tumor de células de la granulosa juvenil maligno. La inmunohistoquímica reportó inhibina $B$ positiva. Se concluyó como estadio IIIA2. Esta es una neoplasia rara con un comportamiento variable. El diagnóstico preciso está basado en los estudios histológicos e inmunohistoquimica.
\end{abstract}

Palabras clave: Tumor de células de la granulosa, Tumor de células de la granulosa juvenil, Ascitis, Diagnóstico.

\section{SUMMARY}

Granulose cell tumors represent 2\% of ovarian tumors. They are classified as adult type and juvenile type. The juvenile type is associated with precocious pseudopuberty, irregular menstruation or nonspecific symptoms such as abdominal pain and palpable mass. More than 95\% are confined to the ovary. A case of a 31-year-old patient is described. The patient consulted for 6-month evolution of increased abdominal volume and weight loss. Right ovary tumor was diagnosed. Tumor excision by gynecological laparotomy with positive frozen cut biopsy and surgical staging were performed. Pathological anatomy reported stromal tumor, and sexual cords suggestive of malignant juvenile granulose cell tumor. Immunohistochemistry reported positive inhibin B. It was concluded as stage IIIA2. This is a rare neoplasm with variable behavior. Accurate diagnosis is based on histological and immunohistochemical studies.

Key words: Granulosa, Cell tumor, Juvenile granulosa cell tumor, Ascites, Diagnosis.

\section{INTRODUCCIÓN}

Los tumores de células de la granulosa (TCG) del ovario, son tumores raros del estroma y cordones sexuales, que abarcan solo el $2 \%$ de todos los tumores

\footnotetext{
${ }^{1}$ Ginecólogo Oncólogo, Ginecólogo Obstetra. Caracas, Venezuela. ${ }^{2}$ Cirujano Oncólogo, Ginecólogo Oncólogo. Jefe del Servicio de Ginecología Oncológica, Servicio Oncológico Hospitalario. Caracas, Venezuela. ${ }^{3}$ Adjunto del Servicio de Anatomía Patológica, Servicio Oncológico Hospitalario. Caracas, Venezuela. ${ }^{4}$ Residente de Postgrado de Ginecología Oncológica, Servicio Oncológico Hospitalario. Caracas, Venezuela.
}

de ovario (1). Estos se clasifican en dos grupos según sus características clínico-patológicas: tipo juvenil y adulto (2). El tumor de células de la granulosa del adulto (TCGA) es el tipo más común y representa casi el $95 \%$ de todos los TCG, por lo general, están presentes en mujeres mayores de 40 años (3). Por el contrario, el tumor de células de la granulosa juvenil (TCGJ), representa menos del $5 \%$ de los TCG y ocurren principalmente en niñas prepúberes y en mujeres menores de 30 años (4).

Los TCGJ son tumores de ovario hormonalmente 
activos. Las mujeres jóvenes suelen tener pseudoprecocidad isosexual. Las mujeres mayores y premenopáusicas se presentan con menorragia o amenorrea o con síntomas inespecíficos, como dolor abdominal o pélvico, distensión o una masa palpable. La torsión o ruptura del tumor causa síntomas abdominales agudos, raramente hirsutismo o virilización, o secundariamente producción de hormonas esteroideas (5-7).

El estadio de la enfermedad es el factor pronóstico más importante y el comportamiento es mucho más agresivo en la etapa avanzada que en el TCGA (8, 9). El TCGJ generalmente se limita al ovario en el momento del diagnóstico y tiene un buen pronóstico. Las pacientes con tumor roto, citología peritoneal positiva o diseminación tumoral extraovárica, tienen un curso clínico agresivo con resultados desfavorables y con mayor riesgo de recurrencia $(10,11)$.

Debido a la rareza de los TCGJ, especialmente en estadios avanzados, a continuación, se reporta un caso, con ascitis masiva como primer signo de presentación, y revisión de la literatura publicada.

\section{HISTORIA CLÍNICA}

Paciente de 31 años de edad, sin antecedentes patológicos, quien presentó aumento de volumen de circunferencia abdominal, asociada a pérdida de peso (10-15 kg) de 6 meses de evolución; acudió a médico en centro foráneo, donde le realizaron un ultrasonido abdominal y pélvico y evidenciaron un tumor multilobulado de ovario izquierdo y ascitis masiva, por lo cual fue referida al Servicio Oncológico Hospitalario (SOH). Al examen físico: Abdomen globoso, predominantemente a expensas de líquido ascítico, signo de oleada positivo, se palpó masa tumoral sólida, de $22 \mathrm{~cm}$ de diámetro, por encima de la sínfisis púbica, no dolorosa a la movilización. Tacto vaginal: Vagina normotérmica, normoelástica, cuello posterior, corto, orificio cervical externo (OCE) rasgado transversalmente, útero en anteversoflexión (AVF), intrapélvico, anexos no palpables, fondos de saco abombados. Tacto rectal: Ambos parametrios laxos. Exámenes paraclínicos: Marcadores tumorales: CA-125: 389,4 Ui/ml, alfafetoproteina, antígeno carcinoembrionario, CA-19-9 y subunidad beta de hormona gonadotropina coriónica (BHCG), todos dentro de límites normales.

El ultrasonido abdominopélvico reportó útero en AVF, de forma, tamaño y bordes normales, con presencia de lesión de ocupación de espacio (LOE) heterogéneo, multilobulado, de alta relación ovárica izquierda, con áreas de vascularidad central y periférica, dimensiones: 25 x $20 \mathrm{~cm}$; ovario derecho no visualizado, ascitis de gran volumen. En la tomografía abdominopélvica con doble contraste, se observó, lesión de aspecto heterogéneo de gran volumen (24 x 18,5 cm), predominantemente sólida, con múltiples septos en su interior, de origen pélvico y alta relación ovárica izquierda, con presencia de ascitis masiva (Figura 1 y 2). Estudios endoscópicos digestivos: sin alteraciones

Se discutió el caso en reunión del servicio de Ginecología Oncológica y se planteó realizar

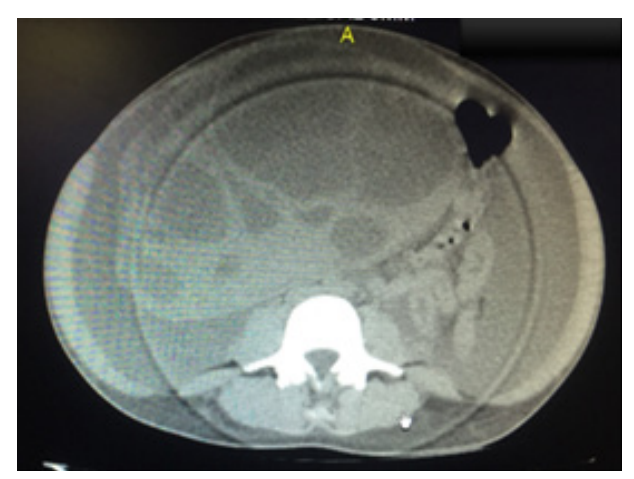

Figura 1: Corte axial de tomografía de abdomen y pelvis con doble contraste, donde se aprecia lesión de aspecto heterogéneo de gran volumen, predominantemente sólida con múltiples septos en su interior, correspondiente a tumor ovárico con presencia de ascitis. 


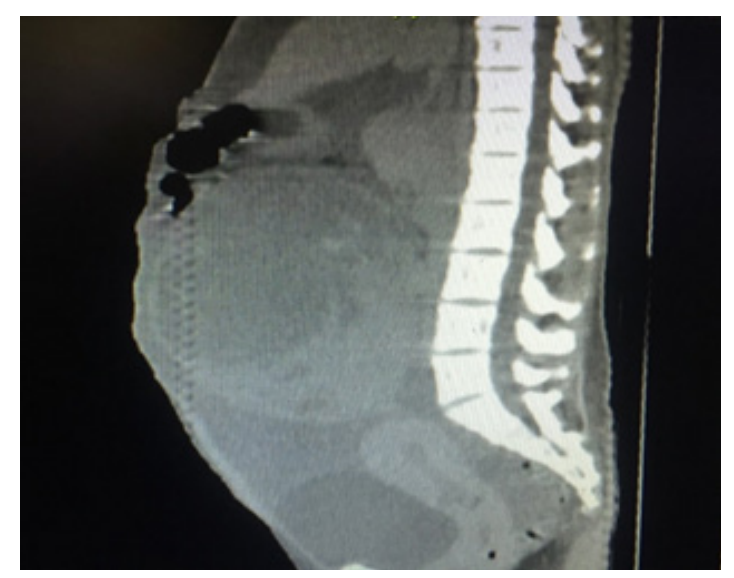

Figura 2: Tomografía de abdomen y pelvis, corte sagital

laparotomía ginecológica, con exéresis del tumor, con estudio histológico por corte congelado. Durante el acto quirúrgico se realizó incisión media infra-, paray supraumbilical. Abordaje de cavidad abdominal, se evidenció: ascitis (5 litros), tumor de ovario izquierdo de $30 \times 20 \mathrm{~cm}$, superficie lisa, regular, multilobulado, de contenido mixto, predominantemente sólido, útero de $8 \times 6 \mathrm{~cm}$, de superficie regular, ovario derecho de $4 \times 3 \times 2 \mathrm{~cm}$; no se palparon adenopatías pélvicas, ni paraaórticas, ni implantes peritoneales (Figuras 3,4 y 5). El corte congelado fue reportado positivo para malignidad, se realizó histerectomía total con ooforosalpingectomía derecha, linfadenectomíapélvica (iliaco - obturatriz), linfadenectomía paraaórtica inframesentérica, omentectomia infracólica, toma de muestras de fondo de saco anterior y posterior, ambas correderas parietocólicas y ambos hemidiafragmas. La paciente evolucionó satisfactoriamente egresando a las 72 horas posteriores a la cirugía.

La biopsia definitiva reportó: ovario izquierdo compatible con tumor del estroma y cordones sexuales sugestivo del TCGJ maligno, invasión vascular presente, con compromiso de la superficie ovárica (Figura 6). Muestras peritoneales, líquido ascítico y ganglios iliacos derechos, con células neoplásicas; el resto de las piezas operatorias evaluadas, sin evidencia de malignidad. Estudio de inmunohistoquímica reportó: inhibina B positivo. En reunión de servicio se concluyó como TCGJ estadio IIIA2, refiriéndose al servicio de oncología médica, para tratamiento adyuvante.

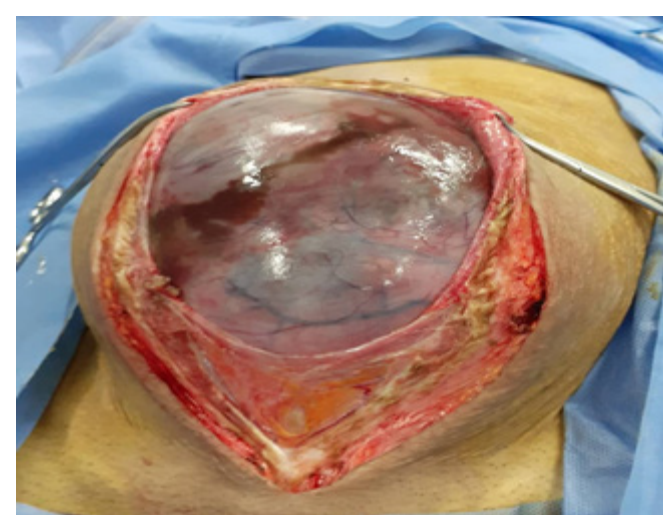

Figura 3: Tumor de ovario izquierdo durante realización de laparotomía

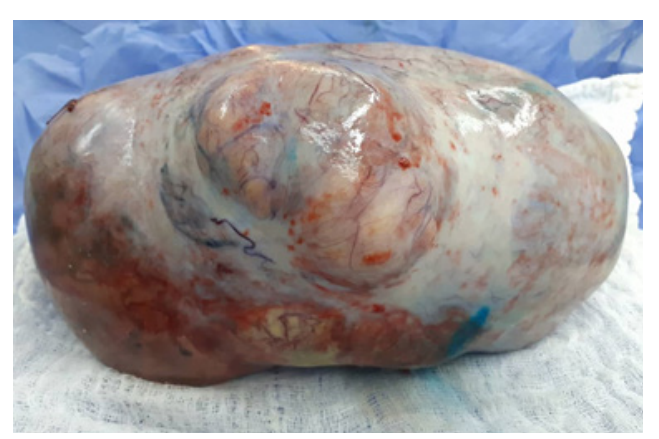

Figura 4: Espécimen quirúrgico de tumor de células de la granulosa Juvenil

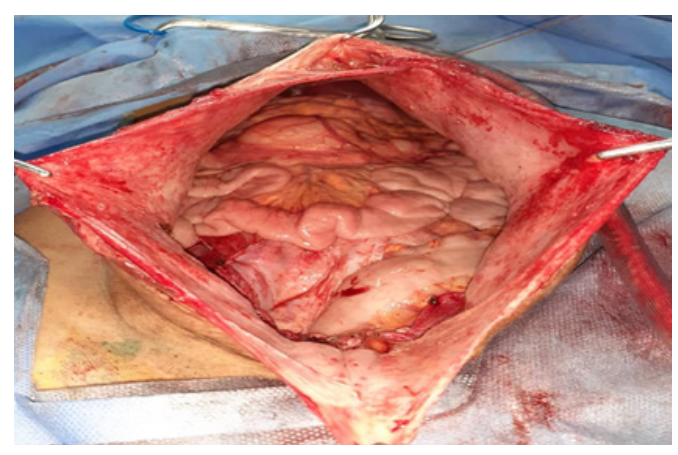

Figura 5: Cavidad abdominal posterior a resección de masa tumoral 


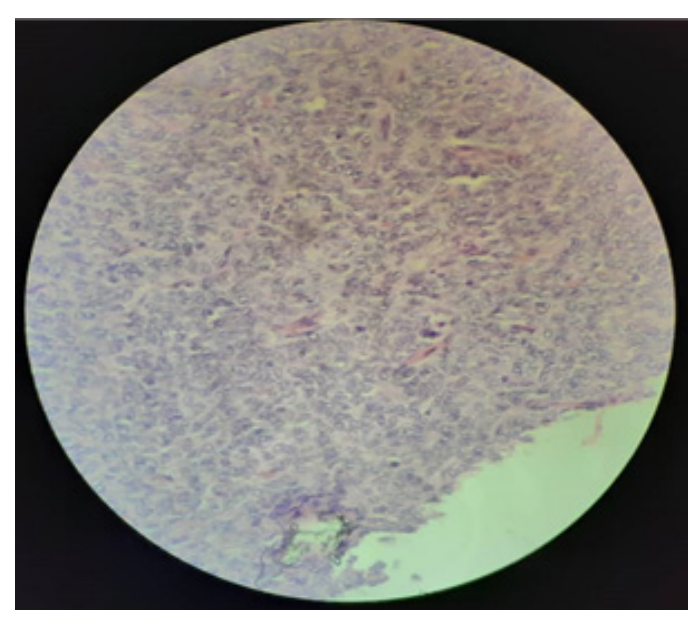

Figura 6: Imagen microscópica a 40X de TCGJ

\section{DISCUSIÓN}

Los TCG del ovario, son tumores poco frecuentes del estroma y cordones sexuales, representando del $1 \%$ al $5 \%$ de todos los tumores ováricos (12). Estos tumores son divididos en TCGA y TCGJ, reflejando no solamente la típica edad de presentación, sino también, las diferentes características histológicas y la historia natural (4). El TCGA es el tipo más común, representa cerca del $95 \%$ de los TCG, se presenta usualmente en mujeres mayores de 40 años (3). Por el contrario, menos del $5 \%$ de estos tumores, son TCGJ y ocurren principalmente en prepúberes y mujeres menores de 30 años, con una edad promedio de 13 años. En una serie, $97 \%$ de los casos se presentaron antes de los 30 años de edad (4). Igualmente, Karalök y cols. (13), reportaron como edad promedio de presentación, los 22 años. A diferencia de lo encontrado en este caso, en el que la edad de presentación fue de 31 años.

Como los TCGJ son hormonalmente activos, la mayor parte de las pacientes presentan características tales como sangrado genital irregular, distención abdominal con dolor, aumento de las mamas, desarrollo de vello púbico y axilar y cambios somatoesqueléticos $(14,15)$. Sin embargo, en este caso, la paciente solo presentó aumento de volumen de la circunferencia abdominal, condicionado por la ascitis masiva y el tumor ovárico. Se encontró el reporte de un caso similar con ascitis masiva, como primer signo de presentación de TCGJ en una adolescente (16).

Los TCG producen inhibina, la cual ha sido utilizada como un marcador sérico en el control de progresión y recurrencia de la enfermedad, desde su uso clínico inicial, a finales de la década de los 80 , la mayoría de los estudios relacionados con la inhibina, incluyeron pacientes con TCGA $(17,18)$. Igualmente se ha confirmado la utilidad de la inhibina B en la detección y control de TCGJ, la cual no pudo realizarse en esta paciente, previo a la cirugía. Los niveles séricos de BHCG y alfafetoproteina, también son importantes para excluir TCG (19); sin embargo, en el presente caso, se encontraban sin alteraciones. Por el contrario, el CA-125, el cual es un marcador tumoral de utilidad en el cáncer epitelial de ovario, en este caso, se encontraba elevado.

Generalmente, la mayoría de los pacientes con TCGJ, son diagnosticados en estadio I, tumor limitado al ovario, y usualmente tienen un comportamiento clínico benigno después de su escisión. Una pequeña proporción se presenta con enfermedad en estadios avanzados II-IV, más difíciles de tratar y con peor pronóstico (20). En el presente caso, el estudio histopatológico reportó, además del ovario, muestras peritoneales, liquido ascítico y ganglios ilíacos derechos con células neoplásicas; constituyendo un estadio IIIA2. El estudio inmunohistoquímico posterior, resultó positivo para inhibina $\mathrm{B}$, la cual es un parámetro de gran utilidad para el diagnóstico de TCGJ.

El estadio al momento del diagnóstico, es el factor pronóstico más importante. En el caso de estadios avanzados, la citorreducción completa, es el objetivo fundamental del tratamiento. En pacientes jóvenes 
con enfermedad confinada a un ovario, la cirugía conservadora pudiera ser considerada $(21,22)$. En el presente caso, se realizó la cirugía estadificadora para cáncer de ovario, tal como se describió anteriormente. Sin embargo, a pesar de que las metástasis a ganglios linfáticos son poco frecuentes, en pacientes con tumores estromales y de los cordones sexuales del ovario, se puede no incluir la linfadenectomía en la cirugía, pero en este caso se realizó, debido a que el corte congelado fue reportado como positivo para malignidad, sin ningún otro detalle, por lo que no se podía excluir una histología de origen epitelial.

La quimioterapia adyuvante, es recomendada en pacientes a partir de estadio IC a IV en TCG, lo cual contribuye a una remisión más duradera $(20,23)$. Esta paciente, después de la cirugía, fue referida al servicio de Oncología Médica, para recibir quimioterapia adyuvante. Aunque las más grandes series en la literatura informan que no se esperarían recurrencias después de tres años del tratamiento, se ha reportado recurrencias tardías $(10,24)$. Por esta razón el seguimiento debe ser por largos periodos, se piensa que la inhibina $\mathrm{B}$, es un marcador de confianza durante el periodo de seguimiento (24).

\section{CONCLUSIÓN}

Los TCGJ son poco frecuentes, con un comportamiento variable, debiendo considerarse como diagnóstico diferencial en pacientes jóvenes con ascitis masiva asociada a tumor de ovario, el diagnóstico preciso se realiza siempre basado en los estudios histológicos y de inmunohistoquímica, constituyendo el estadio al momento del diagnóstico el factor pronóstico más importante. Se requiere un enfoque individualizado al manejar a estas pacientes.

\section{REFERENCIAS}

1. Moid FY, Jones RV. Granulosa cell tumor and mucinous cystadenoma arising in a mature cystic teratoma of the ovary: A unique case report and review of literature.
Ann Diagn Pathol. 2004; 8(2):96-101. doi: 10.1053/j. anndiagpath.2004.01.007. PMID: 15060888.

2. Scully RE. Ovarian tumors. A review. Am J Pathol [Internet]. 1977 [consultado 10 de junio de 2020]; 87(3):686-720. PMID: 194486; PMCID: PMC2032143. Disponible en: https://pubmed.ncbi. nlm.nih.gov/194486/

3. Roth LM. Recent advances in the pathology and classification of ovarian sex cord-stromal tumors. Int J Gynecol Pathol. 2006; 25(3):199-215. doi: 10.1097/01. pgp.0000192271.22289.e6. PMID: 16810055.

4. Young RH. Sex cord-stromal tumors of the ovary and testis: their similarities and differences with consideration of selected problems. Mod Pathol. 2005; 18 Suppl 2:S81-98. doi: 10.1038/modpathol.3800311. PMID: 15502809.

5. Cornejo KM, Young RH. Adult granulosa cell tumors of the testis: a report of 32 cases. Am J Surg Pathol. 2014; 38(9):1242-1250. doi: 10.1097/ PAS.0000000000000216. PMID: 24705318.

6. van Meurs HS, van Lonkhuijzen LR, Limpens J, van der Velden J, Buist MR. Hormone therapy in ovarian granulosa cell tumors: a systematic review. Gynecol Oncol. 2014; 134(1):196-205. doi: 10.1016/j. ygyno.2014.03.573. Epub 2014 Apr 5. PMID: 24713548.

7. Shim SH, Lee SJ, Kim DY, Kim J, Kim SN, Kang $\mathrm{SB}$, et al. A Long-term follow-up study of 91 cases with ovarian granulosa cell tumors. Anticancer Res [Internet]. 2014 [consultado 10 de junio de 2020]; 34(2):1001-1010. PMID: 24511046. Disponible en: https://pubmed.ncbi.nlm.nih.gov/24511046/

8. Calaminus G, Wessalowski R, Harms D, Göbel U. Juvenile granulosa cell tumors of the ovary in children and adolescents: results from 33 patients registered in a prospective cooperative study. Gynecol Oncol. 1997; 65(3):447-452. doi: 10.1006/gyno.1997.4695. PMID: 9190974.

9. Young RH, Dudley AG, Scully RE. Granulosa cell, Sertoli-Leydig cell, and unclassified sex cord-stromal tumors associated with pregnancy: a clinicopathological analysis of thirty-six cases. Gynecol Oncol. 1984; 18(2):181-205. doi: 10.1016/0090-8258(84)90026-x. PMID: 6735262.

10. Young RH, Dickersin GR, Scully RE. Juvenile granulosa cell tumor of the ovary. A clinicopathological analysis of 125 cases. Am J Surg Pathol. 1984; 8(8):575-596. doi: 10.1097/00000478-198408000-00002. PMID: 6465418 . 


\section{TUMOR DE CÉLULAS DE LA GRANULOSA JUVENIL DE OVARIO. PRESENTACIÓN INUSUAL. REPORTE DE UN CASO}

11. Zaloudek C, Norris HJ. Granulosa tumors of the ovary in children: a clinical and pathologic study of 32 cases. Am J Surg Pathol. 1982; 6(6):503-512. doi: 10.1097/00000478-198209000-00002. PMID: 7149091.

12. Outwater EK, Wagner BJ, Mannion C, McLarney JK, Kim B. Sex cord-stromal and steroid cell tumors of the ovary. Radiographics. 1998; 18(6):1523-1546. doi: 10.1148/radiographics.18.6.9821198. PMID: 9821198.

13. Karalök A, Taşçı T, Üreyen I, Türkmen O, Öçalan $\mathrm{R}$, Şahin $\mathrm{G}$, et al. Juvenile granulosa cell ovarian tumor: clinicopathological evaluation of ten patients. J Turk Ger Gynecol Assoc. 2015; 16(1):32-34. doi: 10.5152/jtgga.2015.15207. PMID: 25788847; PMCID: PMC4358309.

14. Bús D, Buzogány M, Nagy G, Vajda G. Rare virilizing granulosa cell tumor in an adolescent. Mol Clin Oncol. 2017; 6(1):88-90. doi: 10.3892/mco.2016.1084. Epub 2016 Nov 18. PMID: 28123736; PMCID: PMC5245144.

15. Carleton C, Houghton OP, McCluggage WG. Juvenile granulosa cell tumor arising in ovarian adenosarcoma: an unusual form of sarcomatous overgrowth. Hum Pathol. 2015; 46(4):614-619. doi: 10.1016/j. humpath.2014.12.010. Epub 2015 Jan 7. PMID: 25656930.

16. Ma L, Zhang L, Zhuang Y, Ding Y, Chen J. A rare case report of ovarian juvenile granulosa cell tumor with massive ascites as the first sign, and review of literature: Case report and review of literature. Medicine (Baltimore). 2018; 97(25):e10916. doi: 10.1097/MD.0000000000010916. PMID: 29923976; PMCID: PMC6023667.

17. Lappöhn RE, Burger HG, Bouma J, Bangah M, Krans $\mathrm{M}$, de Bruijn HW. Inhibin as a marker for granulosacell tumors. N Engl J Med. 1989; 321(12):790-793. doi: 10.1056/NEJM198909213211204. PMID: 2770810.
18. Geerts I, Vergote I, Neven P, Billen J. The role of inhibins $\mathrm{B}$ and antimüllerian hormone for diagnosis and followup of granulosa cell tumors. Int J Gynecol Cancer. 2009; 19(5):847-855. doi: 10.1111/IGC.0b013e3181a702d1. PMID: 19574772.

19. Malati T. Clinical relevance of AFP, its molecular variants and HCG in hepatoblastoma and childhood germ cell tumors. Clin Biochem. 2014; 47(9):734. doi: 10.1016/j.clinbiochem.2014.05.035. Epub 2014 May 20. PMID: 24854689.

20. Schneider DT, Calaminus G, Wessalowski R, Pathmanathan R, Harms D, Göbel U. Therapy of advanced ovarian juvenile granulosa cell tumors. Klin Padiatr. 2002; 214(4):173-178. doi: 10.1055/s-200233183. PMID: 12165898.

21. Colombo N, Parma G, Zanagnolo V, Insinga A. Management of ovarian stromal cell tumors. J Clin Oncol. 2007; 25(20):2944-2951. doi: 10.1200/ JCO.2007.11.1005. PMID: 17617526.

22. Schumer ST, Cannistra SA. Granulosa cell tumor of the ovary. J Clin Oncol. 2003; 21(6):1180-1189. doi: 10.1200/JCO.2003.10.019. PMID: 12637488.

23. Powell JL, Kotwall CA, Shiro BC. Fertility-sparing surgery for advanced juvenile granulosa cell tumor of the ovary. J Pediatr Adolesc Gynecol. 2014; 27(4):e8992. doi: 10.1016/j.jpag.2013.10.001. Epub 2014 Mar 19. PMID: 24656706.

24. Nishida M, Jimi S, Haji M, Hayashi I, Kai T, Tasaka H. Juvenile granulosa cell tumor in association with a high serum inhibin level. Gynecol Oncol. 1991; 40(1):90-94. doi: 10.1016/0090-8258(91)90094-1. PMID: 1989922.

Recibido el 7 de julio 2020 Aprobado el 22 de octubre de 2020 